\title{
A REMARK ON 1-QUASICONFORMAL MAPS
}

\author{
P. TUKIA and J. VÄISÄLÄ
}

1. Introduction. It is well known that if $n \geqq 3$, every 1 -quasiconformal map of a domain $D \subset R^{n}$ is the restriction of a Möbius transformation. For $C^{3}$-maps this was already proved by Liouville in 1850 . The general result is due to Gehring [ $\mathrm{Ge}_{1}$ ] and Rešetnjak [Re]. Their proofs are very deep; a more elementary proof has recently been given by Bojarski and Iwaniec [BI]. Mostow [Mo, (12.2)] pointed out that the case $D=R^{n}$ is much easier; another proof for this case has been given by Gehring $\left[\mathrm{Ge}_{2}\right]$. The purpose of this note is to give a new and simple proof for this special case. It is based on the compactness properties of quasiconformal maps and on the fact that the 1-quasiconformal maps of $R^{n}$ form a group. It is also valid for $n=2$.

2. Notation. For $x \in R^{n}$ and $r>0$ we let $S(x, r)$ denote the sphere $\left\{y \in R^{n}:|y-x|=r\right\}$.

3. Lemma. Let $f: R^{n} \rightarrow R^{n}$ be a homeomorphism such that the image of each sphere $S(x, r)$ is a sphere $S\left(f(x), r_{x}\right)$. Then $f$ is a similarity.

Proof. Let $x, y \in R^{n}$ with $|x-y|=2 r>0$, and let $z=(x+y) / 2$. Consider the sphere $S_{0}$ of radius $r / 2$ which touches the spheres $S_{1}=S(x, r)$ and $S_{2}=S(x, 2 r)$ at $z$ and $y$. Since $f S_{0}$ touches $f S_{1}$ and $f S_{2}, f(z)$ lies on the line segment $f(x) f(y)$. Since $f S(z, r)$ is a sphere centered at $f(z), f(z)=(f(x)+f(y)) / 2$. Hence $f$ preserves the midpoint of every line segment. By iteration and continuity, this implies that $f$ is affine on every line. For each line $L$, there is thus a number $\lambda_{L}>0$ such that $|f(a)-f(b)|=\lambda_{L}|a-b|$ for all $a, b \in L$. Moreover, if the lines $L$ and $M$ intersect, $\lambda_{L}=\lambda_{M}$. It follows that $\lambda_{L}=\lambda$ is independent of $L$.

4. Theorem. Let $n \geqq 2$ and let $f: R^{n} \rightarrow R^{n}$ be 1-quasiconformal. Then $f$ is a similarity.

Proof. By the preceding lemma, it suffices to show that $f$ maps every sphere $S(x, r)$ onto a sphere centered at $f(x)$. With the aid of auxiliary similarity maps, we may assume that $x=0=f(x)$, that $r=1$, that $f\left(e_{1}\right)=e_{1}$, and that the open unit ball $B^{n}$ is contained in $f B^{n}$. Let $W$ be the family of all 1-quasiconformal maps $g: R^{n} \rightarrow R^{n}$ such that $g(0)=0, g\left(e_{1}\right)=e_{1}$, and $B^{n} \subset g B^{n}$. Since $W$ is a closed nonempty normal family [Vä, 19.4, 21.3, 37.4], there is $h \in W$ for which

$$
m\left(h \bar{B}^{n}\right)=\max \left\{m\left(g \bar{B}^{n}\right): g \in W\right\}=M<\infty .
$$


It suffices to show that $M=m\left(\bar{B}^{n}\right)$. If $M>m\left(\bar{B}^{n}\right), \bar{B}^{n}$ is a proper subset of $h \bar{B}^{n}$, and hence $h \bar{B}^{n}$ is a proper subset of $h h \bar{B}^{n}$, which implies $m\left(h h \bar{B}^{n}\right)>M$. Since $h h \in W$, this is a contradiction.

5. Remark. The preceding theorem is also trivially true for $n=1$, if we, as usual, interpret the $K$-quasisymmetric functions $f: R^{1} \rightarrow R^{1}$ as one-dimensional $K$ quasiconformal maps. On the other hand, the same proof gives the following more general result, which is nontrivial also for $n=1$. We allow the possibility that a quasiconformal map is sense-reversing.

6. Theorem. Let $n \geqq 1$, let $K \geqq 1$, and let $G$ be a group of $K$-quasiconformal maps of $R^{n}$ such that $G$ contains all similarity maps. Then $G$ is precisely the group of all similarity maps of $R^{n}$.

In the proof, we may assume that $G$ is closed, replacing it by $\bar{G}$.

Actually, it is sufficient to assume that $G$ contains a group $S$ of similarities such that for each pair of distinct points $x, y \in R^{n}$ there is $g \in S$ such that $g(0)=x$, $g\left(e_{1}\right)=y$. The proof shows that every element of $G$ is then a similarity.

\title{
References
}

[BI] BojaRski, B., and T. IwANIEC: Another approach to Liouville theorem. - Math. Nachr. 107, 1982, 253-262.

[Ge ${ }_{1}$ ] Gehring, F. W.: Rings and quasiconformal mappings in space. - Trans. Amer. Math. Soc. 103, 1962, 353-393.

[Ge ${ }_{2}$ ] Gehring, F. W.: Quasiconformal mappings. - Complex analysis and its applications, vol. 2. International Atomic Energy Agency, Vienna, 1976, 213-268.

[Mo] Mostow, G. D.: Quasi-conformal mappings in $n$-space and the rigidity of hyperbolic space forms. - Inst. Hautes Études Sci. Publ. Math. 34, 1968, 53-104.

[Re] REŠEtNJAK, Ju. G.: Stability of conformal mappings in multi-dimensional spaces. - Sibirsk. Mat. Ž. 8, 1967, 91-114 (Russian).

[Vä] VÄISÄLÄ, J.: Lectures on $n$-dimensional quasiconformal mappings. - Lecture Notes in Mathematics 229. Springer-Verlag, Berlin-Heidelberg-New York, 1971.

\author{
University of Helsinki \\ Department of Mathematics \\ SF--00100 Helsinki 10
}

Finland 